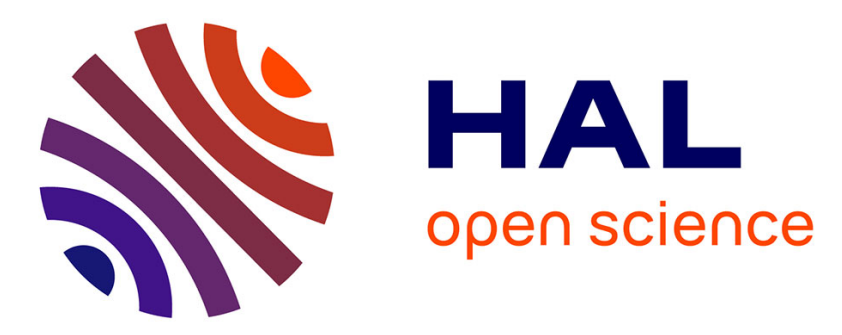

\title{
Three-Dimensional Fluorescence In Situ Hybridization in Mouse Embryos Using Repetitive Probe Sequences
}

\author{
Walid W. Maalouf, Tiphaine Aguirre-Lavin, Laetitia Herzog, Isabelle
} Bataillon, Pascale Debey, Nathalie Beaujean

\section{- To cite this version:}

Walid W. Maalouf, Tiphaine Aguirre-Lavin, Laetitia Herzog, Isabelle Bataillon, Pascale Debey, et al.. Three-Dimensional Fluorescence In Situ Hybridization in Mouse Embryos Using Repetitive Probe Sequences. Fluorescence in situ Hybridization (FISH), 659 (4), Springer, pp.401-408, 2010, Methods in Molecular Biology, 10.1007/978-1-60761-789-1_31 . hal-02610638

\section{HAL Id: hal-02610638 \\ https://hal.science/hal-02610638}

Submitted on 17 May 2020

HAL is a multi-disciplinary open access archive for the deposit and dissemination of scientific research documents, whether they are published or not. The documents may come from teaching and research institutions in France or abroad, or from public or private research centers.
L'archive ouverte pluridisciplinaire HAL, est destinée au dépôt et à la diffusion de documents scientifiques de niveau recherche, publiés ou non, émanant des établissements d'enseignement et de recherche français ou étrangers, des laboratoires publics ou privés. 


\title{
Three-Dimensional Fluorescent In Situ Hybridisation in Mouse Embryos
}

Walid E. Maalouf ${ }^{1,2}$, Tiphaine Aguirre-Lavin ${ }^{1}$, Laetitia Herzog ${ }^{1}$, Isabelle Bataillon ${ }^{1}$, Pascale Debey ${ }^{1}$ and Nathalie Beaujean ${ }^{1}$

${ }^{1}$ INRA, UMR 1198 Biologie du Développement et Reproduction, F-78350 Jouy en Josas, France

${ }^{32}$ Present Address: QMRI, 47 Little France Crescent, University of Edinburgh, Edinburgh, UK

Contact : Dr Walid Maalouf <walidmaalouf@ hotmail.com>, Tel. +33 (0)1 34652903 / Fax: 2909

\begin{abstract}
A common problem in research laboratories that study the mammalian embryo is the limited supply of live material. For this reason, new methods are always developed and existing methods for cells in culture are adapted to suit this peculiar experimental model. Three-Dimensional fluorescent in situ hybridisation (3D-FISH) is an important tool to study how genomic sequences are positionned in nuclei without altering their $3 \mathrm{D}$ organisation. This provides vital information about the distribution of specific sequences in relation to nuclear substructures such as chromocenters and nucleoli. In this manuscript, we will present a detailed description on how to carry out 3D-FISH in the early preimplantation mouse embryo.
\end{abstract}

Keywords: 3D; FISH; in situ; embryo; mouse; development. 


\section{Introduction}

Fluorescent in situ hybridisation (FISH) is a cytogenetic technique that provides researchers with a direct way to visualize and map the genetic material in individual cells, including specific genes or parts of specific chromosomes(1). This technique has become important in preimplantation genetic diagnosis (PGD). Often families with known or suspected genetic diseases want to know more about their putative child's conditions before proceeding with full term pregnancy. These concerns can be addressed by FISH to diagnose for a number of diseases such as Prader-Willi syndrome, Angelman syndrome, 22q13 deletion syndrome, chronic myelogenous leukemia, acute lymphoblastic leukemia, Cri-du-chat, Velocardiofacial syndrome, and Down syndrome. On the other hand, many researchers are now studying the nonrandom spatial distribution of chromosomes and genes within the nuclear space with 3D-FISH approaches as the organization of the genome inside the interphase nucleus seems to be a central determinant of genome functions and that knowing the sequence of a genome is insufficient to understand its physiological function (3). Using interphasic nuclei with preserved 3D shape, one can indeed determine the threedimensional arrangements of chromosomes, providing detailed information about the compartmentalization of chromosomes into discrete territories (4). It is also possible to locate gene-rich versus gene-poor regions and evaluate the dynamic interactions of specific genomic sequences with other nuclear components (5). For instance, we recently demonstrated using the 3D_FISH method that the organisation of constitutive heterochromatin in the early mouse embryo is associated with their development to term (6).

According to the experimental design, probes used for FISH will bind only to parts of the chromosomes or to specific genomic sequences with which they show a high degree of sequence homology. In this chapter, continuous repetitions of short monomer probes will be used to study two repetitive sequences from the heterochromatic region of the nucleus, centromeric and pericentromeric repeats, also known as minor and major satellites. The probes will be directly tagged with different fluorochromes for simultaneous detection. Thereby, by using multiple probes labeled with different fluorochromes, scientists are able to label each chromosome in its own unique color (the result is known as a spectral karyotype) but also several genomic sequences at the same time, within the same nuclei (7). In all cases, fluorescence microscopy will be used to locate the FISH signal in the sample.

Unlike cells in culture, mouse embryos are surrounded by glycoprotein rich membrane known as the zona pellucida (8) and therefore they do not attach to the plates when cultured in vitro. Moreover, the nuclei of early pre-implantation embryos are larger than those of differentiated cells which makes it more difficult to preserve the three-dimensional structure during the procedure. In this chapter we will describe such multi-color FISH with centromeric and pericentromeric probes in mouse embryos, using both direct and indirect detection.

\section{Materials}

\subsection{Embryos Preparation}

1. M2 culture medium embryo-tested (Sigma-Aldrich, Saint-Quentin Fallavier, France). Store at $4{ }^{\circ} \mathrm{C}$. 
2. Phosphate Buffered Saline (PBS) tablets (Sigma-Aldrich, Saint-Quentin Fallavier, France) dissolved in distilled water according to manufacturer's instructions, autoclaved and then stored at $4{ }^{\circ} \mathrm{C}$.

3. Glass dishes (Electron Microscopy Sciences, Euromedex, Mundolsheim, France)

4. Thin glass manipulation pipettes for embryos, usually home-made with glass capillaries or Pasteur pipettes.

5. Tyrode's solution (Sigma-Aldrich, Saint-Quentin Fallavier, France) stored in single use aliquots of $1 \mathrm{ml}$ at $-20{ }^{\circ} \mathrm{C}$.

6. Fixative: $20 \%$ paraformaldehyde (PFA) solution, EM grade (Electron Microscopy Sciences, Euromedex, Mundolsheim, France) with adjusted pH ( 7.4) and stored at $4{ }^{\circ} \mathrm{C}$ with light protection (aluminium foil for example). Working solutions of $4 \%$ are prepared by dilution with PBS just before use.

7. SuperFrost Plus slides (VWR, Fontenay-sous-Bois, France).

8. Hydrophobic barrier pen (Sigma-Aldrich, Saint-Quentin Fallavier, France).

\subsection{In Situ Hybridization}

1. Phosphate Buffered Saline (PBS) tablets (Sigma-Aldrich, Saint-Quentin Fallavier, France) dissolved in distilled water according to manufacturer's instructions and autoclaved. Store at $4^{\circ} \mathrm{C}$.

2. Permeabilization stock solution: $10 \%(\mathrm{w} / \mathrm{v}$ in distilled water) Triton $\mathrm{X}-100$ (Sigma-Aldrich, Saint-Quentin Fallavier, France) solution. Store at $4{ }^{\circ} \mathrm{C}$. The $0.5 \%$ working solution is prepared by dilution with PBS just before use.

3. 20X saline-sodium citrate (SSC) buffer (Sigma-Aldrich, Saint-Quentin Fallavier, France) containing $0.3 \mathrm{M}$ sodium citrate and $3 \mathrm{M} \mathrm{NaCl}$ at a $\mathrm{pH}$ around 7.0. The working solutions of $0.1 \mathrm{X}$ and $2 \mathrm{X}$ SSC at a $\mathrm{pH}$ of 6.3 are prepared by diluting the $20 \mathrm{X}$ stock in $\mathrm{PBS}$ and lowering the $\mathrm{pH}$ with the appropriate volume of $\mathrm{HCl}$.

4. Labelled probe: StarFISH pan-centromeric probe Cy3 conjugated ( 200nt; Cambio, Cambridge, UK).

5. Unlabelled probe: consensus 80-nt forward sequence for murine minor satellites biotinylated at its 5' end (Invitrogen, Cergy-Pontoise, France) (9).

6. RNase A (Invitrogen, Cergy-Pontoise, France) stored at $1 \mathrm{M}$ in single use aliquots of $100 \mu \mathrm{l}$ at $-20^{\circ} \mathrm{C}$.

7. Heating block set at $85^{\circ} \mathrm{C}$ (Grant, VWR, Fontenay-sous-Bois, France).

8. Deionized formamide (Molecular Biology Grade; Sigma-Aldrich, SaintQuentin Fallavier, France)

9. Hybridization buffer: $50 \%$ formamide, $10 \%$ dextran sulphate (from $50 \% \mathrm{w} / \mathrm{v}$ stock solution), $1 \mathrm{X}$ Denhardt, $40 \mathrm{mM} \mathrm{NaH} \mathrm{PO}_{4}$ in $2 \mathrm{X} \mathrm{SSC}$, pH 7 (all products from Sigma-Aldrich, Saint-Quentin Fallavier, France). Store in single use aliquots of $1 \mathrm{ml}$ at $-20{ }^{\circ} \mathrm{C}$.

10. Hybridisation incubator with humidified loading tray for slides, set at $37{ }^{\circ} \mathrm{C}$ (Grant Boekel, VWR, Fontenay-sous-Bois, France).

11. Humidified non-transparent box in which you can keep the slides during the different incubation steps.

12. Detection compound for the unlabelled probe: Streptavidin conjugated with Alexa 633 (Molecular Probes, Invitrogen, Cergy-Pontoise, France)

13. Nuclear counterstain: $10 \mu M$ Yo-Pro-1 (Molecular Probes, Invitrogen, CergyPontoise, France).

14. Mounting medium: Citifluor (Biovalley, Marne-la-Vallée, France). 


\section{Glass cover slips 22 x $40 \mathrm{~mm}$ (CML, Nemours, France)}

\section{Methods}

Preimplantation embryos can be collected in vivo just before 3D-FISH or cultured according to standard protocols. However, embryos are known to be very sensitive to culture conditions that can affect development (e.g. cleavage rates, fragmentation and quality of post-implantation) and might alter chromosome/gene positioning. We therefore advise to prepare all materials and equipments in advance and perform mounting of the embryos on slides as quickly as possible.

\subsection{Mounting the Embryos on Slides}

1. Label the slide with name and date, and draw one, maximum two, squares $(1 \times 1$ $\mathrm{cm}$ ) on your slide with the hydrophobic pen (see Note 1). Each square should be allocated to one group of embryos (with 10 to 20 embryos/group) therefore several slides might be required.

2. Prepare culture plates with microdrops $(25-50 \mu \mathrm{l})$ of $\mathrm{M} 2$ solution to rinse the embryos, and keep at room temperature in open air to equilibrate.

3. Defrost on the bench one aliquot of Tyrode's solution per group of embryos (see Note 2).

4. The first step is to denude the embryo by removing the zona pellucida in order to improve access of the probes within the embryos (see Note 3). Take the first group of embryos to be processed and transfer it from the collection / culture medium into the M2 medium. Load the manipulation pipette with fresh Tyrode's solution, and then transfer embryos into a glass dish containing $500 \mu 1$ of Tyrode's solution. Immediately reload the manipulation pipette with fresh Tyrode and then transfer embryos into a second glass dish containing another $500 \mu 1$ of Tyrode's solution. Incubate the embryos for no more than 1.5 minutes (see Note 4, 5).

5. Quickly rinse the embryos in multiple drops of the pre-equilibrated M2 and place them inside the drawn square on the glass slide.

6. Aspirate slowly excess M2 medium in order to fix the embryos onto the glass slide (see Note 6).

7. As soon as the embryos are fixed and M2 removed, add 10-15 $\mu 1$ of $4 \%$ PFA

8. Keep slide(s) in a humidified box at $4^{\circ} \mathrm{C}$, overnight (see Note 7).

\subsection{Hybridization with the Probes}

1. On day 2, gently aspirate the PFA and replace with PBS for 30 minutes to rinse the embryos.

2. Permeabilise the embryos for 30 minutes with $0.5 \%$ Triton X-100 (diluted in PBS just before use).

3. Rinse embryos in PBS then in 2x SSC for 5 minutes each time.

4. To decrease unspecific background, RNAs are digested by incubation in $200 \mu M$ of RNAse diluted in $2 x \mathrm{SSC}\left(\mathrm{pH}\right.$ 6.3) for 30 minutes at $37^{\circ} \mathrm{C}$.

5. Rinse embryos in $2 x \mathrm{SSC}(\mathrm{pH}$ 6.3) then in PBS for 5 minutes each time.

6. Equilibrate in hybridization buffer for 1-2 hr (see Note 8).

7. In a $0.5 \mathrm{ml}$ tube, mix $1 \mu \mathrm{l}$ of each of the probe solutions with $12 \mu \mathrm{l}$ of hybridization buffer and denature at $85{ }^{\circ} \mathrm{C}$ for 10 minutes, and transfer the tube into ice straight after.

8. At the same time, put your glass slides with the embryos (still in the hybridization buffer) on the heating block to denature the DNA at $85{ }^{\circ} \mathrm{C}$ for 10 minutes (see Note 9). 
9. Replace the buffer on the embryos with the hybridization mix and place the slides in the humidified loading tray of the hybridization incubator at $37{ }^{\circ} \mathrm{C}$ for $24 \mathrm{hr}$ (see Note 10).

10. On day 3, several washing steps are required to remove the remaining unbound probes and decrease the unspecific background. First, rinse embryos in prewarmed $\left(37^{\circ} \mathrm{C}\right) 2 \times \mathrm{SSC}$ for $3 \times 5$ minutes at $37^{\circ} \mathrm{C}$. Then in pre-warmed $\left(60^{\circ} \mathrm{C}\right)$ $0.1 \times$ SSC for $3 \times 5$ minutes at $60^{\circ} \mathrm{C}$.

11. Further rinse the embryos at RT in $0.1 x$ SSC then in PBS for 5 minutes each time.

12. The DNA is counterstained with $10 \mu M$ of Yo-Pro-1 in PBS for 15 minutes (see Note 11).

13. Rinse the embryos for $2 x 5$ minutes and post-fix with $2 \%$ PFA for another 5 minutes (post-fixation helps maintaining the fluorescent signal until observation).

14. After removal of the PFA, slowly add one drop of mounting medium, and gently place the coverslip.

15. Seal the borders of the coverslip with nail varnish and keep slide at $4{ }^{\circ} \mathrm{C}$ in the dark until observation with a microscope (see Note 12).

16. An example of the results produced is shown in Fig. 1 (see Note 13).

\section{Notes}

1. Due to the nature of the solutions that are used in this method and that can easily dilute / erase ink (even permanent one), a diamond pencil is usually used to label the slides.

2. Do not use Tyrode's solution more than 15 minutes after defrosting as it looses its activity and becomes ineffective on embryos.

3. When dealing with several groups, we advise to carry out the denudation process for one group at a time as it is a very critical step in this experiment. An extended incubation with the acid will damage the embryos and their nuclei, while a short incubation time might not be enough to remove the zona pellucida completely.

4. This step and all subsequent ones are carried out at room temperature (RT) unless otherwise stated.

5. Under the dissection microscope, you can observe the zona pellucida being degraded by the acid activity of the Tyrode's solution.

6. Even though this step is carried out at room temperature, the light from the microscope produces enough heat to dehydrate the embryos. This must be avoided to preserve the 3D structure of the nuclei. Rehydration can be accomplished by adding M2 medium if the embryos are not yet fixed on the slide .

7. Alternatively, incubate in $4 \%$ PFA for 30 minutes at room temperature and proceed to the next step. 

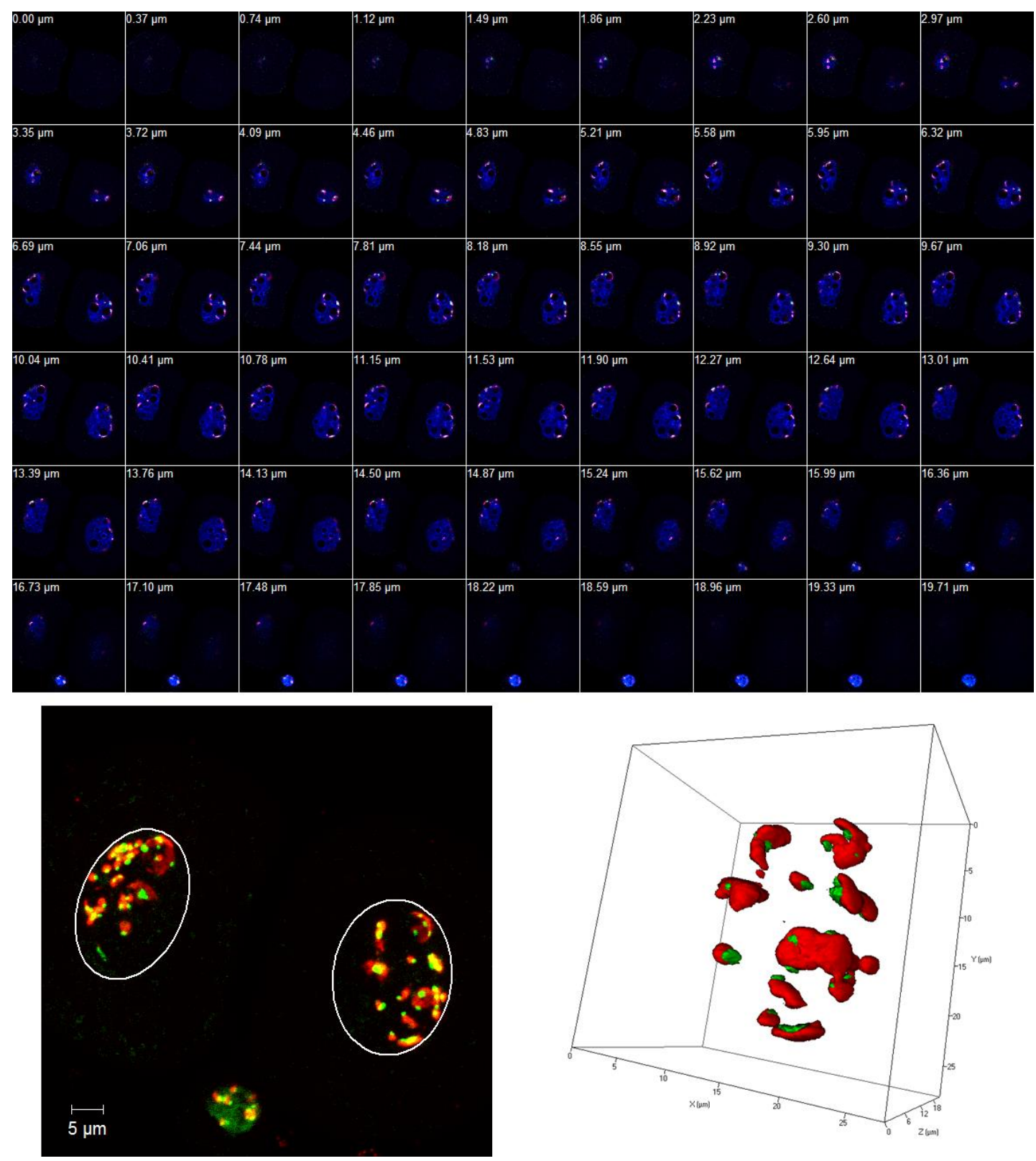

Fig. 1. 3D-FISH on a two-cell stage mouse embryo with the pan-centromeric and centromeric probes counterstained with Yo-Pro-1. The z-serie (upper panel, z-step of $0.37 \mu \mathrm{m}$ ) was acquired on a Zeiss LSM510 confocal with a plan-apochromat $63 \mathrm{x} / 1.4$ oil DIC objective at 488, 543 and $633 \mathrm{~nm}$ wavelenghts sequentially. Blue color was assigned to DNA, red to the pan-centromeric signal and green to the centromeric one to allow better signal vs. background contrast for the biologist. With the LSM browser software the z-serie can be merged to give a single image with the two probes (lower pannel, left) on which each nuclei is underlined by a white cercle. 3D reconstruction of one nucleus from this embryo (lower pannel, right) clearly shows the conserved shape of the nucleus and the so-called «chromocenters » formed by aggregations of major (pan-centromeric probe) and minor (centromeric probe) satellites.

8. It is recommended to switch-on the heating block when equilibration starts in order to reach the desired temperature for the subsequent steps.

9. To prevent the microdrops from evaporating during that step filter paper humidified with formamide $50 \%$ should be placed in the bottom of the tray. 
10. All subsequent steps are carried out in a non-transparent humidified chamber.

11. Other nuclear counterstain, such as propidium iodide, can be used according to the choice of fluorochromes used to label the probes.

12. Confocal or grid microscopy is necessary for three-dimensional imaging. The optimal parameters to obtain the best $3 \mathrm{D}$ reconstruction is to perform Zsectionning at no more than $0.5 \mu \mathrm{m}$ between sections and to take 2-3 additional sections on top and below the limits of the nucleus.

13. If the fluorescent signal is too weak, amplification of the signal may be necessary. Secondary components such as fluorescently-tagged secondary antibodies can be used to provide a more pronounced signal. 


\section{Acknowledgements}

We thank Pierre Adenot for technical assistance on the confocal microscope platform MIMA2 (Microscopie et Imagerie des Microorganismes, Animaux et Elements) and UEAR for animal care. We are also grateful to Eve Devinoy (INRA) and Claire Francastel (Institut Cochin) for their help. This work was supported by INRA grant "Crédits incitatifs PHASE".

\section{References}

1. Halling, K. C., Kipp, B. R. (2007) Fluorescence in situ hybridization in diagnostic cytology. Hum Pathol 38, (8), 1137-44.

2. Sreekantaiah, C. (2007) FISH panels for hematologic malignancies. Cytogenet Genome Res 118, (2-4), 284-96.

3. Foster, H. A., Bridger, J. M. (2005) The genome and the nucleus: a marriage made by evolution. Genome organisation and nuclear architecture. Chromosoma 114, (4), 212-29.

4. Walter, J., Joffe, B., Bolzer, A., Albiez, H., Benedetti, P. A., Muller, S., Speicher, M. R., Cremer, T., Cremer, M., Solovei, I., Towards many colors in FISH on 3D-preserved interphase nuclei. Cytogenet Genome Res 2006, 114, (3-4), 367-78.

5. Kupper, K., Kolbl, A., Biener, D., Dittrich, S., von Hase, J., Thormeyer, T., Fiegler, H., Carter, N. P., Speicher, M. R., Cremer, T., Cremer, M. (2007) Radial chromatin positioning is shaped by local gene density, not by gene expression. Chromosoma 116, (3), 285-306.

6. Maalouf, W. E., Liu, Z., Brochard, V., Renard, J. P., Debey, P., Beaujean, N., Zink, D. (2009) Trichostatin A treatment of cloned mouse embryos improves constitutive heterochromatin remodeling as well as developmental potential to term. BMC Dev Biol 9, 11.

7. Werner, M., Wilkens, L., Aubele, M., Nolte, M., Zitzelsberger, H., Komminoth, P. (1997) Interphase cytogenetics in pathology: principles, methods, and applications of fluorescence in situ hybridization (FISH). Histochem Cell Biol 108, (4-5), 381-90.

8. Wassarman, P. M. (2005) Contribution of mouse egg zona pellucida glycoproteins to gamete recognition during fertilization. J Cell Physiol 204, (2), 388-91.

9. Bouzinba-Segard, H., Guais, A., Francastel, C. (2006) Accumulation of small murine minor satellite transcripts leads to impaired centromeric architecture and function. Proc Natl Acad Sci U S A 103, (23), 8709-14. 